\title{
Gruppo Italiano di Cardiologia Riabilitativa e Preventiva: attività del biennio 2002-2004
}

\author{
Francesco Fattirolli \\ Coordinatore GICR
}

Monaldi Arch Chest Dis 2004; 62: 2, 117-118.

Con il Congresso nazionale di Torino, si conclude il mandato dell'attuale Consiglio Direttivo del GICR eletto nel corso dell'assemblea di Cosenza. Nei due anni appena trascorsi, l'impegno è stato rivolto a sviluppare ed a qualificare ulteriormente $\mathrm{i}$ contenuti e le modalità dell'intervento riabilitativo, unitamente alle competenze professionali degli operatori. Per raggiungere questi obiettivi e per favorire una ulteriore crescita del GICR, sono stati sviluppati programmi culturali, organizzativi ed associativi, cercando di recepire i suggerimenti e le proposte dalle sedi periferiche, che è auspicabile giungano sempre più numerosi nel prossimo futuro.

Al centro del progetto di questo biennio vi è stata - come si prevedeva nel documento programmatico "l'affermazione della cultura della Cardiologia Riabilitativa, dall'appropriatezza della valutazione all'efficacia dell'intervento globale", unitamente alla promozione di iniziative scientifiche, di formazione ed aggiornamento, rivolte a tutte le componenti professionali della Cardiologia Riabilitativa, e lo sviluppo degli organi ufficiali rappresentati della rivista e dal sito web.

Sarebbe di scarsa utilità fare una elencazione puntuale di ciò che è stato fatto, e dell'impegno che è stato dedicato alla vita del GICR. Mi limiterò soltanto ad alcune annotazioni, con particolare riguardo a ciò che dovrà ulteriormente essere sviluppato.

Lo Studio Gospel, non occorre sottolinearlo, rappresenta attualmente il maggiore "investimento" della cardiologia riabilitativa italiana sul piano scientifico. Dovrà essere mantenuto con rigorosa puntualità e completezza il lavoro fino a conclusione del follow-up, perché solo allora potranno essere analizzati i risultati e verificati gli obiettivi che lo studio si prefiggeva. Il Gospel è strategico per il GICR e per tutta la cardiologia italiana, per la necessità di disporre di dati di outcome sull'intervento strutturato ed intensivo di prevenzione secondaria.

Le Commissioni hanno portato a termine il lavoro loro affidato, con la produzione di documenti, tutti pubblicati sulla nostra rivista:

1) linee guida per l'intervento psicologico in cardiologia riabilitativa; 2) raccomandazioni per la riabilitazione del paziente molto anziano; 3) raccomandazioni per la riabilitazione nel paziente diabetico; è in fase di ultimazione il lavoro della commissione che ha elaborato le raccomandazioni per l'esecuzione ed interpretazione del test cardiopolmonare.

La Formazione ha rappresentato uno dei punti centrali del programma di attività, come momento di approfondimento, di verifica e di confronto delle personali conoscenze con quelle di esperti, in funzione anche della domanda in continua crescita, sia tra i cardiologi che tra tutto il personale sanitario dell'équipe riabilitativa.

Il GICR ha partecipato ad iniziative di formazione e aggiornamento presentate da varie sedi regionali sia nella forma di corsi tradizionali che di master per medici o per il personale non medico, oltre ad organizzare un "corso avanzato" - nell' ambito del programma formativo ANMCO - articolato in sessioni tematiche con la partecipazione di esperti di altri settori (cardiochirurgo, elettrofisiologo, emodinamista, diabetologo, geriatra, etc.) che hanno consentito di ampliare il confronto e sviluppare la discussione sulle prospettive di più stretta collaborazione con la cardiologia riabilitativa.

Il censimento delle strutture e l'analisi dei percorsi riabilitativi (progetto ISYDE) nei Centri Italiani - per l'infarto ed il post-operato - pur con qualche disomogeneità, hanno fornito elementi utili per conoscere la realtà di riferimento nazionale e per descrivere, per la prima volta, la modalità di lavoro dei Centri, nella diversità delle proprie realtà geografiche, istituzionali ed organizzative. Tuttavia il "progetto ISYDE" non è concluso ma anzi costituisce la base di un sistema di "reporting" periodico per aggiornare e "rinnovare" il data-base, al fine di realizzare entro breve tempo uno strumento aggiornato continuativamente e disponibile on-line per gli operatori e per le Agenzie sanitarie regionali.

I Referenti Regionali rivestono un ruolo strategico per le attività del Gruppo. Le differenze delle realtà legislative, i piani sanitari, l'accreditamento, i rapporti talora conflittuali con altri professionisti, richiedono una attività diretta e continuativa dei Referenti, per conoscere le diversità, rendere possibile il confronto e consentire di elaborare strategie comuni. Sono state effettuate periodiche riunioni collegiali e stabilite modalità di comunicazione continua con la Segreteria del GICR, al fine di rendere efficace questa collaborazione. Un ringraziamento non formale a quanti fino ad ora hanno dato il proprio fattivo contributo ed un augurio a coloro che in futuro saranno chiamati a questo nuovo impegno.

L'integrazione tra le differenti componenti professionali è stato uno dei programmi centrali di questo biennio. La multidisciplinarietà è uno dei punti di forza della Cardiologia Riabilitativa: l'integrazione di conoscenze, di esperienze e di capacità propositive è il mezzo più adeguato per elaborare e rendere efficaci programmi comuni di lavoro. Per dare concretezza a questo impegno sono stati formalizzati i Gruppi delle Aree (Psicologi, Infermieri, Fisioterapisti, Dietisti) che porteranno il contributo delle rispettive professioni con l'obiettivo di integrare le com- 
petenze, sviluppare iniziative di aggiornamento, rendere omogenei i percorsi assistenziali etc.

Rapporti con le altre Società e Associazioni Scientifiche. Il GICR per l'insieme di attività che promuove o sostiene, e per la capacità dimostrata dai Centri di Riabilitazione di partecipare agli studi scientifici, risulta uno dei più vitali nel panorama della Cardiologia italiana. Il costante rapporto con l'ANMCO, ma anche con la SIC, con l'ANCE e l'ARCA, con le Società di Cardiochirurgia, di Emodinamica etc., deve essere un obiettivo da consolidare per mettere in atto un'interazione dialettica ed efficace finalizzata alla diffusione della "cultura" della cardiologia riabilitativa.

Nel corso dell'ultimo anno si è realizzata una sinergia con l'Area Prevenzione dell'ANMCO, che ha tutte le potenzialità per svilupparsi ulteriormente nel prossimo futuro. Vi sono state iniziative comuni, quali un Minimaster e un Simposio al Congresso nazionale di cardiologia dell'ANMCO, ma soprattutto sono state costituite due commissioni congiunte per la stesura di documenti di consenso in tema di prevenzione secondaria e dell'alto rischio, che hanno presentato le proprie conclusioni alla III Conferenza Nazionale sulla Prevenzione Cardiovascolare, organizzata nell'Aprile 2004 da Istituto Superiore di Sanità, Area Prevenzione ANMCO, Heart Care Foundation e GICR.

Anno del Cuore. La necessità di garantire una presenza efficace del GICR per la diffusione dei contenuti che sono patrimonio "originale" della Cardiologia Riabilitativa all'interno - o a fianco - delle varie manifestazioni che ogni anno si svolgono in Italia su temi di prevenzione cardiovascolare, si è realizzata anche con la partecipazione alla "Alleanza per il Cuore". Unitamente alle principali Società, Associazioni e Fondazioni cardiologiche nazionali oltre che alle Associazioni degli Utenti, prime fra tutte il CoNaCuore, sono stati forniti i contributi che hanno portato il Ministero della Salute a promuovere l'iniziativa "2004 Anno del Cuore".

Gli organi Ufficiali del GICR. Il "Monaldi Archives for Chest Diseases" e il Sito web "gicr.it" hanno vissuto uno sviluppo, sia per qualità che per quantità, che è sotto gli occhi di tutti: l'invito è di adoperarsi affinché la rivista si arricchisca sempre più di contributi scientifici, ma anche di opinioni, corrispondenze, notizie dai Centri e dalle Regioni, che facciano conoscere a tutti le diverse realtà della cardiologia riabilitativa italiana e possano stimolare un dibattito costruttivo così da rappresentare il terreno di confronto di idee ed esperienze differenti.

Il Sito web deve sempre più diventare strumento di lavoro quotidiano: per le informazioni che mette a disposizione sia per gli Associati che per l'utente "laico"; per la possibilità di rendere disponibili notizie, aggiornamenti, programmi formativi per i cardiologi e le altre figure professionali; per la gestione del data base continuamente aggiornato dei Centri nazionali di cardiologia riabilitativa.

Il GICR oggi. Quanto si riesce a realizzare nel corso di due anni è sempre inferiore ai propositi che si fanno all'inizio del mandato; se il lavoro è stato efficace ed ha prodotto risultati saranno gli Associati ed il mondo cardiologico a valutarlo piuttosto che quanti hanno ricevuto l'incarico di dedicare il proprio impegno a questo compito. Non è quindi il momento di bilanci, ma di alcune considerazioni sulla vita del GICR.

La nostra Associazione è in grado di guardare con ottimismo al proprio percorso, anche se non mancano criticità su cui confrontarsi sia all'interno che all'esterno. Mi riferisco alla percezione che non tutti abbiamo la stessa visione dei contenuti e delle modalità organizzative della cardiologia riabilitativa alla luce della attuale realtà assistenziale e legislati$\mathrm{va}$, in un contesto epidemiologico che è in continuo, e talvolta vertiginoso, cambiamento. Nella multiforme rappresentazione della riabilitazione cardiologica, convivono esperienze ed interessi diversi tra loro: sono diversi i modelli organizzativi e gli obiettivi "politici" che le strutture e gli operatori si prefiggono, sono presenti talvolta logiche economiche che spingono a privilegiare un modello e conseguentemente a penalizzarne un altro. In più, sono frequenti più o meno latenti tentativi dall'esterno di egemonizzare spazi che sono propri della cardiologia riabilitativa. Occorre pertanto mantenere vivo un confronto costruttivo, all'interno del quale arrivare alla identificazione di una "piattaforma" comune sulla quale costruire il "modello base di riferimento" della cardiologia riabilitativa degli anni duemila.

Ritengo che il GICR possieda al suo interno tutte le capacità e la maturità per realizzare questa ulteriore crescita, attraverso la piena presa di coscienza di tutte le specificità che per tradizione e per cultura sono al suo interno. Serve però impegno, rigore scientifico, capacità propositiva e di collaborazione, talvolta anche intransigenza. È necessario cercare con insistenza, in primis nell'interesse dei malati, di collaborare con i cardiochirurghi, con gli emodinamisti, con gli elettrofisiologi, etc.; stabilire progetti con i Medici del territorio; rendere disponibili - alle altre strutture ed operatori della cardiologia - le conoscenze operative e tecniche che sono patrimonio unico della cardiologia riabilitativa: la multidimensionalità e multidisciplinarietà della valutazione e dell'intervento, il modello del "percorso" terapeutico, il counseling e l'educazione, il progetto preventivo, l'esercizio fisico come strumento di cura, prevenzione e mantenimento della salute. Questa, nel suo insieme, è la nostra "tecnologia" originale.

L'esperienza professionale ed umana realizzata con i componenti del Consiglio Direttivo che termina il proprio mandato, ha consentito una conoscenza reciproca che va ben oltre l'impegno nel lavoro associativo. Sento il desiderio di far partecipe chi legge della mia sincera gratitudine nei riguardi di coloro che hanno condiviso con me questo impegno e con il loro sostegno mi hanno permesso di ricoprire il ruolo di coordinatore in questi due anni: la saggezza di Carmine Chieffo, la concretezza di Salvatore Pirelli, la razionalità di Roberto Tramarin, la fantasia di Romualdo Belardinelli, l'entusiasmo di Margherita Vona, la ponderazione di Carlo Vigorito, il buon senso di Lello Griffo, la propositività di Stefano Urbinati, la dedizione di Gigi Temporelli, l'impegno di Luisa Briolotti.

E grazie, infine, a tutti coloro - e sono tanti - che hanno dato il proprio contributo con grande disponibilità ed a quanti ci hanno fatto riflettere anche soltanto con una critica o un suggerimento. 